\section{Down's Anomaly}

2nd ed. By G. F. Smith and J. M. Berg. (Pp. ix + 348; 102 figures + 113 tables. £12.50) Edinburgh, London and New York: Churchill Livingstone. 1976.

A decade has passed since the first edition of Penrose and Smith's book on Down's Anomaly. In 1973, Lionel Penrose died. After Langdon Down, he had made the major contribution to our understanding of mongolism. The new edition pays tribute to both Penrose and Langdon Down and is written by George Smith, co-author of the first edition, and another Penrose devotee, Joe Berg. There have been many advances since the 1965 edition. Chromosome banding techniques have identified the chromosomes involved in translocation Down's. Studies on abortion material have indicated how frequently trisomy 21 occurs in first trimester abortions. The word amniocentesis does not appear in the index of the 1965 edition but is now a part of every woman's vocabulary. These changes are included in the new edition. Penrose campaigned for other changes. He would have preferred the word mongolism to have disappeared by now. It is, however, still deeply entrenched in everyday professional usage and is perhaps marginally better than 'Kalmuck idiocy' and the 'ill-finished' or 'unfinished' child.

With the birth incidence of Down's anomaly still as high as 1 in 660 live births, paediatricians, obstetricians, community physicians, and geneticists need a good source of reference on the subject. This second edition provides this. The chapter on vital statistics, cytology, and aetiology will answer all the practical questions about recurrence risks. The research worker or clinician cannot but be intrigued by the early onset of what seems to be Alzheimer's disease in mongols, the occurrence of severe infantile and transient forms of leukaemia, and the altered immune mechanism. The references are more than generous.

The only serious omission is in the section on 'social and educational considerations'. One looked for 'official' refutation of the still prevalent 'Madam, in my experience, your child will never learn to talk. Put him in an institution and forget you ever had him'. Despite this lack, Lionel Penrose would have been well satisfied with this second edition of his book.

\section{BARAITSER}

\section{Principles of Genetic Counselling}

By Edmond A. Murphy and Gary A. Chase. (Pp. 380; 14 Figures + 17 Tables. £13.75.) Year

Book Medical Publishers; Chicago; London: Lloyd-Luke. 1975.

Murphy and Chase have written an attractively individualistic account of genetic counselling. They are concerned with the philosophical and mathematical principles underlying counselling rather than with offering a detailed practical guide. The book will be hard reading for most medical practitioners and, indeed, for many medical geneticists, but rewarding for both. Even the experienced genetic counsellor will find himself taking a second look at some of the assumptions by which he practises the art.

A chapter on probability theory defines terms which are often used imprecisely. 'Likelihood', for example, the authors suggest, is best used for events that have already occurred. The chapter on counselling for Mendelian conditions is perhaps over-elaborated, but draws attention, for example, to the substantial affect on the estimate of risk for X-linked conditions provided by the unaffected males already present in the family. The chapter on ancillary methods sets out the important topic of how to combine information on risks from two independent sources-for example, from pedigree information and creatine kinase estimations in severe X-linked muscular dystrophy. A chapter on two locus systems is perhaps of mainly theoretical interest at present. The chapter on 'multilocal' systems, which follows the usual lines, does not appear to have seen the relation of the Edwards and Falconer models. The term 'multilocal' is proposed for a character which is under the control of several gene loci, reserving the term 'polygenic' for the situation in which the several gene loci are of about equal importance. The chapter on empirical risk counselling is sound in principle, but the text suggests some lack of experience of the realities of the more common malformations. The risk after several pregnancies affected by neural gene malformations is not 'comparatively small'. A table of risks for anencephaly by maternal age and social class has little relevance to genetic counselling in the usual situation, where parents have had one or more affected children or a parent himself is affected.

Genetic counselling is essentially a service to individual couples, but the authors are to be con- 
gratulated on also discussing in a final chapter the eugenic implications of genetic counselling. The effects of mutation rate and altered fitness for unilocal conditions are set out in several Tables (there appear to be inconsistencies in some of these which will need correction in a later edition). The authors note that the entire impact of fresh recessive mutation can be borne by a mild reduction in the planned fitness of heterozygotes. They might have made the further point that, in the future, it will probably not be the practice to select against individual recessives, but to evaluate the total number of recessives that an individual couple carry between them.

On positive eugenics the authors rightly stress the difficulty of knowing just which qualities will be socially desirable several generations hence. They also stress that, in a democratic society, individual parents have to be judges in their own case and themselves make the decision about the likely genetic worth of their children. Many would take the view that such decisions are, nevertheless, a moral responsibility that every couple must shoulder.

Cedric CARTER

Experimental Biology and Medicine Cytogenetic aspects of Malignant Transformation

By N. B. Atkin. Vol. 6. Series Editors: A., Wolsky, D. J. Pizzarella, G. V. Sherbet, J. Steiner. (Pp. viii + 168; 21 Figures + 9 Tables. DM. 74.) Basel: $S$. Karger. 1976.

The author has provided, within the confines of this short monograph, a comprehensive and reasonably up-to-date review of many aspects of chromosomes and malignancy.

The early chapters survey the kinds of chromosome abnormalities found in malignant cells, chromosome breaking agents, and constitutional chromosome abnormalities (including the 'breakage syndromes') which may predispose to cancer, and there is a brief consideration of premalignancy. The greater part of the text is taken up with details of the chromosome changes found in the leukaemias and various solid tumours of man. There are then short chapters dealing with tumours of other animals and plants, various in vitro methods for studying malignancy, and some general conclusions. The book is by no means profusely illustrated, but the figures are generally adequate and well chosen.

Dr Atkin describes his book as a progress report and it is unfortunate that, even now, little cytogenetic information is available from some tumour types, which is not of prebanding vintage. This is particularly true of many solid tumours, where technical difficulties in obtaining good cell suspensions and $\stackrel{\mathbb{D}}{\overparen{D}}$ adequate numbers of mitoses mean that it will be some $\stackrel{\mathbb{P}}{-}$ time before much systematically collected banding $\overrightarrow{\vec{F}}$ data become available. On the other hand, bone marrow is a very suitable tissue for chromosome? studies, and consequently a great deal has been learnt $\frac{\bar{\sigma}}{\bar{c}}$ recently of the precise cytogenetic changes which take $\frac{\sigma}{\widetilde{\sigma}}$ place in both malignant and premalignant haemato- $\propto$ logical disorders: much of this information may be found here (there are references cited up to the middle $\vec{\circ}$ of 1975).

The main shortcoming of this monograph is that it $\vec{\omega}$ is very largely descriptive. However, with nearly 800 ? references, it may be recommended as a valuable $\bar{\Xi}$ background for all workers in the field of cancer cyto- $\frac{\vec{G}}{\mathrm{G}}$ genetics.

B. R. REEVES $\ddot{-}$

Chemical Mutagens. Principles and Methods for their Detection

Vol. 4. Edited by Alexander Hollaender. (Pp. xviii $+\stackrel{\text { g }}{2}$ 339; Figures + Tables. \$35.94.) New York:

Plenum Press. 1976.

Recent years have seen a massive upsurge of interest in the potential genetic hazard to man of environmental chemicals. This has been reflected in the first three volumes of Hollaender's series Chemical Mutagens. The latest addition is not for those who seek an introduction to or a justification for genetic $\stackrel{\square}{\mathbb{Q}}$ toxicology - earlier volumes will suffice for that-but $\overrightarrow{\vec{P}}$ it contains much of value and relevance to those $\frac{3}{3}$ interested in environment or industrial medicine. $\bar{J}$ The past two years have seen strong support for the somatic mutation theory of cancer or, to be more precise, for a strong correlation between carcino- $\overline{0}$ genicity and the ability of an agent to damage the $\dot{0}$ hereditary material DNA. This correlation is accepted 3 . by most of the contributors to Volume 4 except $\delta$ Lijinsky who, in a characteristically idiosyncratic chapter on $\mathrm{N}$-nitroso compounds, neverthelesso manages to be both informative and challenging. Though he might not accept this, we probably do nowo know where to look for answers to the problems of chemical carcinogenicity raised by Lijinsky such aso organ and species specificity, even if we do not yet 0 know what they are.

Medicines are, almost by definition, biologically ${ }_{\sigma}^{\omega}$ active substances and it is not surprising that a number of them turn out to be mutagenic, usually after meta- bolic activation occurring within the body. Legator, $\$$ Zimmering, and Connor contribute a useful chapter on ways of detecting mutagenic activity in the blood ${ }_{-}^{-}$ or urine of exposed animals and patients. Many 